\title{
Electron-beam irradiation as an alternative to preserve nutritional, chemical and antioxidant properties of dried plants during extended storage periods
}

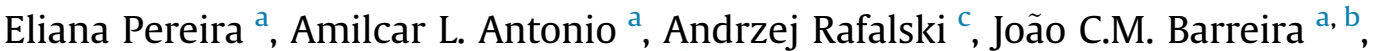 \\ Lillian Barros ${ }^{a}$, M. Beatriz P.P. Oliveira ${ }^{\text {b }}$, Isabel C.F.R. Ferreira ${ }^{\text {a, }}{ }^{*}$ \\ a Centro de Investigação de Montanha (CIMO), ESA, Instituto Politécnico de Bragança, Campus de Santa Apolónia, 1172, 5300-253 Bragança, Portugal \\ ${ }^{\mathrm{b}}$ REQUIMTE/LAQV, Faculdade de Farmácia, Universidade do Porto, Rua Jorge Viterbo Ferreira n. ${ }^{\circ} 228,4050-313$ Porto, Portugal \\ ${ }^{\mathrm{C}}$ Centre for Radiation Research and Technology, Institute of Nuclear Chemistry and Technology, Dorodna str. 16, 03-195 Warsaw, Poland
}

\section{A R T I C L E I N F O}

\section{Article history:}

Received 5 January 2017

Received in revised form

11 March 2017

Accepted 22 April 2017

Available online 27 April 2017

\section{Keywords:}

Electron-beam irradiation

Chemical/nutritional composition

Antioxidant activity

Extended storage

\begin{abstract}
A B S T R A C T
According to current market demands, there is an increasing need for improved conservation methodologies. In addition to an extension in shelf-life, food products should preserve their compositional integrity and bioactive properties throughout storage time. Irradiation technology has been progressively considered as a feasible conservation technology. Electron-beam irradiation, in particular, might be predominantly suitable to be applied in food products with reduced thickness, such as aromatic and medicinal plants. In this study, the effects of e-beam irradiation on chemical, nutritional and antioxidants parameters of different plant species were evaluated. To assess the potential of this technology over extended periods, plant samples were stored for the first time up to a maximum of 18 months. Despite some heterogeneity among the effects produced in each plant species, electron-beam treatment attenuated the reduction of individual compounds (primarily, free sugars, organic acids, tocopherols and polyunsaturated fatty acids) verified in non-irradiated samples, showing its potential as an alternative conservation technology.
\end{abstract}

(c) 2017 Elsevier Ltd. All rights reserved.

\section{Introduction}

Food irradiation is being progressively categorized as a versatile, efficient, safe, secure and highly effective conservation technique. Reasons underlying this classification are related with its ability to provide stability to nutritious foods, besides preserving healthpromoting properties during longer storage periods (Cabo Verde et al., 2010; Hunter, 2000; Roberts, 2014).

From a legal standpoint, safety and efficiency of food irradiation have been recognized by authorities such as the World Health Organization (WHO), the International Atomic Energy Agency (IAEA), and the Food Agriculture Organization FAO (Farkas \& MohácsiFarkas, 2011; Farkas, 2006) regarding three types of ionizing radiation: gamma radiation, X-rays and electron-beam (EU, 1999).

Gamma radiation derives from the spontaneous emission of ${ }^{60} \mathrm{Co}$ or ${ }^{137} \mathrm{Cs}$ isotopes; X-rays are produced by the impact of

\footnotetext{
* Corresponding author.

E-mail address: iferreira@ipb.pt (I.C.F.R. Ferreira).
}

accelerated electrons on a metallic target, which deaccelerates the electrons, emitting electromagnetic radiation by a physical phenomenon designated as "bremsstrahlung" (literally, braking radiation); electron-beam (e-beam) radiation is produced by accelerating a stream of electrons, focusing them into beams that can be directed to food products on the conveyor belt (mega electron volt) (Farkas, 2008). Despite being able to reach higher energies than X-rays or gamma rays, the maximum energy in food processing is limited to $10 \mathrm{MeV}$, due to technical and safety reasons (EU, 1999). In addition, e-beam irradiation requires short time, is less expensive and does not produce nuclear waste (Wei et al., 2014).

The specific characteristics of e-beam irradiation make it particularly suitable for food products with low density and small size, such as aromatic and medicinal herbs. These plants might suffer chemical and biological contaminations throughout their production process (harvesting, drying, packaging and storage), causing spoilage, quality deterioration and consequently economic loss (Darfour, Agbenyegah, Ofosu, Okyere, \& Asare, 2014). 
Considering their wide use in pharmaceutical, food and cosmetic industry (Katušn-Razěm, Novak, \& Razěm, 2001; Haleem, Salem, Fatahallah, \& Abdelfattah, 2014), attaining a feasible conservation and decontamination treatment for these matrices might represent good technological advantages.

Besides its decontaminating effectiveness, e-beam irradiation, such as any other preserving treatment, should be able to maintain (or ideally improve) as much characteristics as possible of the treated product (Migdal \& Owczarczyk, 1998). Therefore, it is mandatory to evaluate if the chemical profiles (especially considering individual compounds), physical parameters (particularly those related to the product appearance) and bioactive properties (e.g., antioxidant activity) are globally maintained throughout storage time (Nagy, Solar, Sontag, \& Koenig, 2011).

Herein, the nutritional, chemical and antioxidant properties of aromatic herbs with highly disseminated use (A. citrodora Paláu, M. officinalis L., M. melissophyllum L. and M. pipperita L.) were evaluated in non-irradiated and e-beam irradiated samples submitted to a maximum storage of 18 months (Pereira, Antonio, Rafalski, et al., 2015). The main purpose was verifying if e-beam treatment had the ability to preserve the initial characteristics of the plant species, validating the process in natural matrices where this extended storage periods were not tested before, thereby providing additional commercial opportunities.

\section{Materials and methods}

\subsection{Samples}

Plant material was obtained from representative species: (Aloysia citrodora Paláu, Melissa officinalis L., Melittis melissophyllum L. and Mentha pipperita L.) as previously described (Pereira, Antonio, Rafalski, et al., 2015; Pereira, Antonio, Barreira, et al., 2015). Samples were analysed immediately after irradiation (0 months) and after storage in a dry place protected from light for 12 and 18 months. For each period, individual sample groups (unirradiated or irradiated with a $10 \mathrm{kGy}$ dose) were analysed.

\subsection{Standards and reagents}

Solvents such as acetonitrile, n-hexane and ethyl acetate (HPLC grade) were purchased from Fisher Scientific (Lisbon, Portugal). Fatty acids methyl ester (FAME, standard 47885-U) mixture was purchased from Sigma (St. Louis, MO, USA), as also other individual standards such as trolox, tocopherol ( $\alpha$ - and $\delta$-isoforms), sugar ((D(-)-fructose, D-(+)-sucrose, D-(+)-glucose, D- $(+)$-trehalose and D$(+)$-raffinose pentahydrate) and organic acids (oxalic acid, quinic acid, malic acid, citric acid and fumaric acid). Tocol (50 mg/mL), $\beta$ tocopherol and $\lambda$-tocopherol were purchased from Matreya (Pleasant Gap, PA, USA). Water was treated in a Milli-Q water purification system (TGI Pure Water Systems, Greenville, SC, USA).

\subsection{Irradiation treatment}

In this process three types of dosimeters were used (a standard dosimeter, a graphite calorimeter, and two routine dosimeters: Gammachrome YR and Amber Perspex) (from Harwell Company; Oxfordshire, UK). Electron-beam irradiation was conducted at the Institute of Nuclear Chemistry and Technology, Warsaw, Poland (Pereira, Antonio, Rafalski, et al., 2015). Irradiation was performed in an e-beam irradiator of $10 \mathrm{MeV}$ of energy with a pulse duration of $5.5 \mu \mathrm{s}$, a pulse frequency of $440 \mathrm{~Hz}$ and an average beam current of $1.1 \mathrm{~mA}$; the scan width was $68 \mathrm{~cm}$, the conveyer speed was settled to the range $20-100 \mathrm{~cm} / \mathrm{min}$ and the scan frequency was $5 \mathrm{~Hz}$. The estimated absorbed dose for irradiated samples was
$10.09 \mathrm{kGy}$, with a maximum uncertainty of $20 \%$. In the Amber Perspex and Gammachrome YR dosimeters, the irradiation dose was estimated by spectrophotometric measurement at $603 \mathrm{~nm}$ and $530 \mathrm{~nm}$, respectively, by comparison with a calibration curve. For the graphite calorimeter the electrical resistance was read and converted in dose according to a calibration curve, obtained during the Quality Control procedures of the irradiation equipment and facility.

\subsection{Nutritional composition}

Protein, fat, carbohydrates and ash content was determined following official procedures (AOAC, 2002). Crude protein content was determined by the macro-Kjeldahl method $(\mathrm{N} \times 6.25)$; crude fat was determined with a Soxhlet apparatus by extracting ( $\approx 12 \mathrm{~h}$ ) a known weight of sample with petroleum ether; ash content was determined by incineration in a muffle furnace $\left(600 \pm 15^{\circ} \mathrm{C}\right)$ until a whitish ash appear; carbohydrates were calculated by difference. The energetic value was calculated according to the equation: En$\operatorname{ergy}(\mathrm{kcal})=4 \times\left(\mathrm{g}_{\text {protein }}+\mathrm{g}_{\text {carbohydrates }}\right)+9 \times\left(\mathrm{g}_{\mathrm{fat}}\right)$.

\subsection{Colour measurement}

Colour parameters were evaluated using a colorimeter (model CR-400, from Konica Minolta Sensing, Inc., Japan), with an adapter for granular materials (model CR-A50). The illuminant $C$ and diaphragm aperture of $8 \mathrm{~mm}$ were used. CIE $L^{*} a^{*} b^{*}$ colour space values were registered using "Spectra Magic Nx" software (version CMS100W 2.03.0006, Konica Minolta, Japan).

\subsection{Chemical composition}

\subsubsection{Sugars}

Free sugars were determined and analysed by HPLC coupled to a refractive index detector (Barros et al., 2013). Dried extracts ( $\approx 1 \mathrm{~g}$ ) were spiked with melezitose (internal standard) (IS, $5 \mathrm{mg} / \mathrm{mL}$ ), and extracted with $40 \mathrm{~mL}$ of $80 \%$ aqueous ethanol at $80{ }^{\circ} \mathrm{C}$ for $30 \mathrm{~min}$. The resulting suspension was centrifuged (Centurion K24OR refrigerated centrifuge, West Sussex, UK) at $15,000 \mathrm{~g}$ for $10 \mathrm{~min}$. The supernatant was concentrated at $60^{\circ} \mathrm{C}$ under reduced pressure and defatted three times with $10 \mathrm{~mL}$ of ethyl ether. After concentration at $40{ }^{\circ} \mathrm{C}$, the solid residues were dissolved in water to a final volume of $5 \mathrm{~mL}$ and filtered through $0.2 \mu \mathrm{m}$ Whatman nylon filters. Compounds were identified by chromatographic comparisons with authentic standards and quantified using the internal standard method. Data were analysed with a Clarity 2.4 Software (DataApex, Prague, Czech Republic).

\subsubsection{Organic acids}

Organic acids were determined following a previously optimized procedure (Barros et al., 2013). Samples ( $\approx 2 \mathrm{~g}$ ) were extracted by stirring with $25 \mathrm{~mL}$ of meta-phosphoric acid $\left(25^{\circ} \mathrm{C}\right.$, $150 \mathrm{rpm}, 45 \mathrm{~min}$ ) and subsequently filtered through Whatman No. 4 paper. Before analysis, samples were filtered again through $0.2 \mu \mathrm{m}$ nylon filters. UFLC-DAD (Shimadzu Corporation, Kyoto, Japan) was used for the analyses, using $215 \mathrm{~nm}$ as preference wavelength. Organic acids were quantified using calibration curves obtained from commercial standards of each compound. Data were analysed with LabSolutions software (Shimadzu Corporation, Kyoto, Japan).

\subsubsection{Tocopherols}

Tocopherols were determined by HPLC coupled to a fluorescence detector (Knauer, Berlin, Germany), using a procedure previously described by Pereira, Barros, and Ferreira (2013). Samples 
(500 mg) were homogenized with $4 \mathrm{~mL}$ methanol by vortex mixing for $1 \mathrm{~min}$. Subsequently, $4 \mathrm{~mL}$ hexane was added and vortexed again $(1 \mathrm{~min})$. Then $2 \mathrm{~mL}$ saturated $\mathrm{NaCl}$ aqueous solution was added, the mixture was homogenized ( $1 \mathrm{~min}$ ), centrifuged (5 min, $4000 \mathrm{~g}$ ) and the clear upper layer was carefully transferred to a vial. Each sample was re-extracted twice with hexane. Combined extracts were taken to dryness under a nitrogen stream, re-dissolved in $2 \mathrm{~mL}$ of $\mathrm{n}$-hexane, dehydrated with anhydrous sodium sulphate, filtered through $0.2 \mu \mathrm{m}$ nylon filters (Whatman) and transferred into a dark injection vial for the analysis. Chromatographic comparisons with standards were made to identify the compounds. Quantification was based on the fluorescence signal response of each standard, using tocol as the internal standard. Data was analysed with Clarity 2.4 Software (DataApex, Prague, Czech Republic).

\subsubsection{Fatty acids}

Fat was extracted with petroleum ether in a Soxhlet apparatus. Fatty acids (obtained after Soxhlet extraction) were methylated with $5 \mathrm{~mL}$ of methanol:sulphuric acid:toluene 2:1:1 (v/v/v), during at least $12 \mathrm{~h}$ in a water bath $\left(50{ }^{\circ} \mathrm{C} ; 160 \mathrm{rpm}\right)$; then $3 \mathrm{~mL}$ of deionized water was added to obtain phase separation; FAME were recovered with $3 \mathrm{~mL}$ of diethyl ether by shaking in vortex, and the upper phase was dehydrated with sodium sulphate anhydrous; finally samples were filtered through $0.2 \mu \mathrm{m}$ nylon filter (Whatman). The analysis was made using gas-liquid chromatography with flame ionization detection (GC-FID) (Pereira et al., 2013).
Identification was made by comparing the relative retention times of FAME peaks from the samples with standards. Results were analysed using CSW 1.7 Software (DataApex, Prague, Czech Republic).

\subsection{Evaluation of antioxidant properties}

\subsubsection{Extracts preparation}

Methanolic extracts were prepared from the dry leaves, stirring the sample ( $\approx 1 \mathrm{~g}$ ) with $30 \mathrm{~mL}$ of methanol $\left(25^{\circ} \mathrm{C}, 150 \mathrm{rpm}, 1 \mathrm{~h}\right)$. The extracts were then filtered through Whatman No. 4 paper and the residues were re-extracted under the same conditions. Combined extracts were concentrated under reduced pressure (rotary evaporator Büchi R-210; Flawil, Switzerland) and re-dissolved in methanol at $10 \mathrm{mg} / \mathrm{mL}$. For infusion preparation, $2 \mathrm{~g}$ of powdered dry leaves were added to $200 \mathrm{~mL}$ of boiling distilled water, left to stand at room temperature for $10 \mathrm{~min}$, and then filtered under reduced pressure, frozen, lyophilized and re-dissolved in distilled water at a final concentration of $10 \mathrm{mg} / \mathrm{mL}$.

\subsubsection{Antioxidant activity}

DPPH radical-scavenging activity, reducing power, inhibition of $\beta$-carotene bleaching, total phenols and total flavonoids were evaluated by colorimetric assays (Pereira et al., 2013). The results were then converted to $\mathrm{EC}_{50}$ values $(\mathrm{mg} / \mathrm{mL})$ by using the graphs of the antioxidant activity percentage or absorbance at $690 \mathrm{~nm}$, in the

Table 1

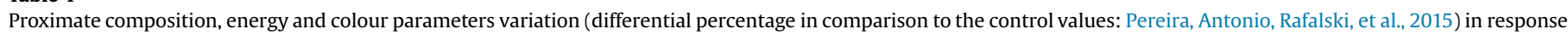
to electron-beam at $10 \mathrm{kGy}$ and storage time. The results are presented as mean \pm standard deviation.

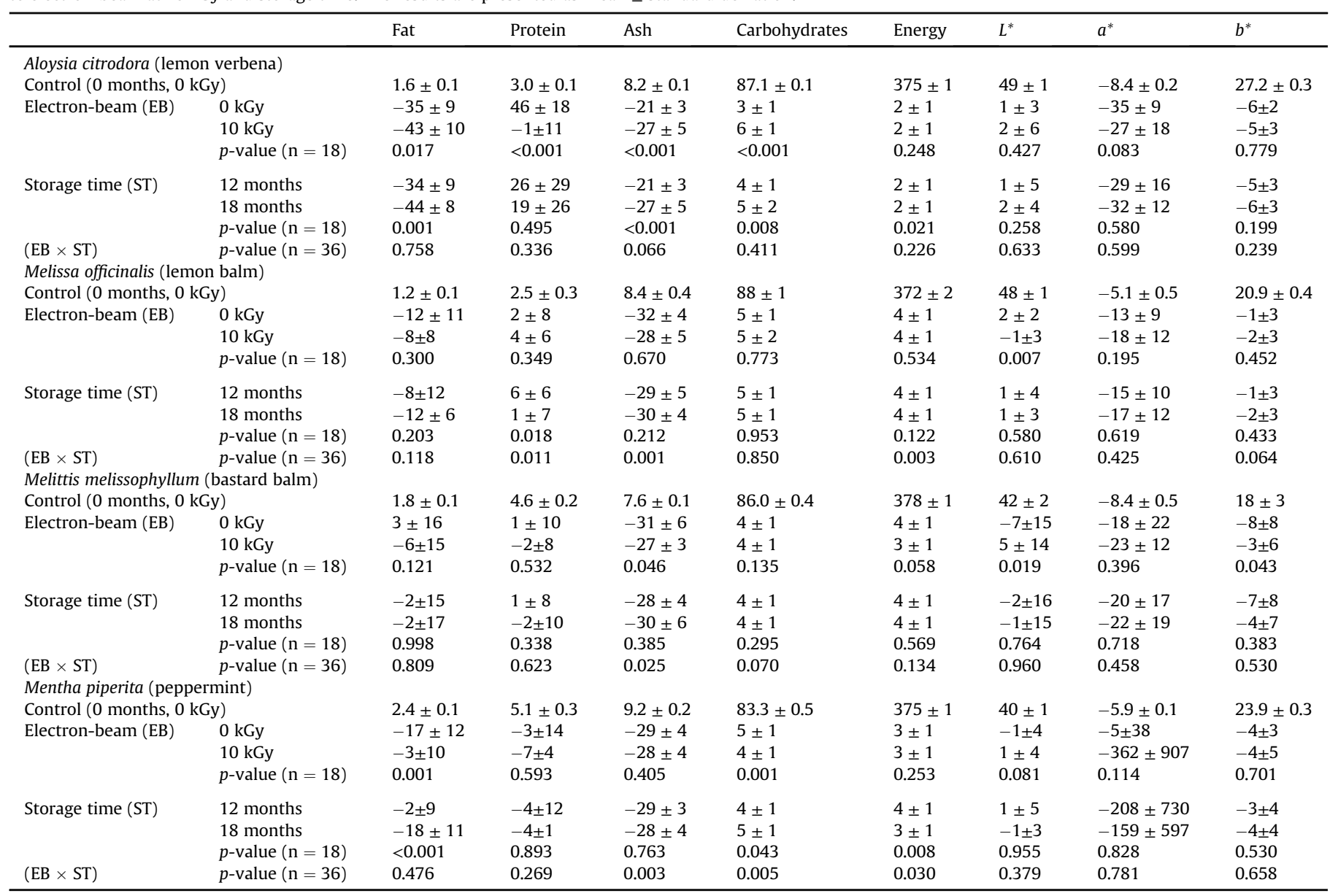


case of reducing power assay, against the extract concentration. Trolox, a water-soluble analogue of vitamin E, was used as a positive control.

\subsection{Statistical analysis}

Three samples (each corresponding to $\approx 40 \mathrm{~g}$ of dried leaves from different plants) were analysed for each of the levels of the assayed factors (irradiation dose, storage time and plant species). Instead of presenting the absolute measured values, data were given as a standardized difference among the values obtained for each treated sample under the same conditions and the respective control ((treated sample value - control value)/control value $\times 100$ ) (Pereira, Antonio, Rafalski, et al., 2015).

Since more than one factor might contribute to the variability of results, the differences among mean values were compared by applying an analysis of variance (ANOVA) with type III sums of squares. The GLM (General Linear Model) procedure of the SPSS ( $v$. 22.0, IBM Corp., Armonk, NY: USA) was used to perform the 2-way ANOVA. The considered factors were "storage time" (ST) and "electron-beam dose" (EB). Besides evaluating the influence of each factor alone, their interaction $(\mathrm{ST} \times \mathrm{EB})$ was also evaluated and, if significant, obtained differences were analysed from the estimated marginal means plots; on the other hand, if the interaction was not statistically significant $(p>0.05)$, means belonging to each factor were compared using the $t$-student test.

Furthermore, principal components analysis (PCA) was applied to gain overall understanding of the most significant effects exerted by EB and ST, as also their correlation with the standardized variation calculated for each analytical parameter. The number of

Table 2

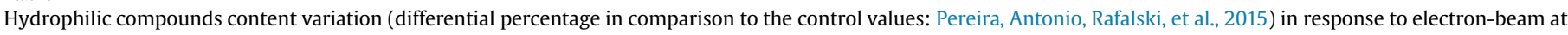
$10 \mathrm{kGy}$ and storage time. The results are presented as mean \pm standard deviation.

\begin{tabular}{|c|c|c|c|c|c|c|c|c|c|c|c|c|}
\hline & & Fructose & Glucose & Sucrose & Trehalose & $\begin{array}{l}\text { Total } \\
\text { sugars }\end{array}$ & $\begin{array}{l}\text { Oxalic } \\
\text { acid }\end{array}$ & Quinic acid & Malic acid & $\begin{array}{l}\text { Shikimic } \\
\text { acid }\end{array}$ & Citric acid & $\begin{array}{l}\text { Organic } \\
\text { acids }\end{array}$ \\
\hline \multicolumn{13}{|c|}{ Aloysia citrodora (lemon verbena) } \\
\hline $\begin{array}{l}\text { Control (0 mont } \\
\text { Electron-beam } \\
\quad \text { (EB) }\end{array}$ & $\begin{array}{l}\text { hs, } 0 \text { kGy) } \\
0 \mathrm{kGy} \\
10 \mathrm{kGy} \\
p \text {-value } \\
(\mathrm{n}=18)\end{array}$ & $\begin{array}{l}1.0 \pm 0.1 \\
-5 \pm 19 \\
-13 \pm 14 \\
0.199\end{array}$ & $\begin{array}{l}1.3 \pm 0.1 \\
-37 \pm 16 \\
-29 \pm 14 \\
0.119\end{array}$ & $\begin{array}{l}7.1 \pm 0.3 \\
-12 \pm 12 \\
-41 \pm 18 \\
<0.001\end{array}$ & $\begin{array}{l}1.2 \pm 0.1 \\
8 \pm 24 \\
-5 \pm 18 \\
0.072\end{array}$ & $\begin{array}{l}10.7 \pm 0.4 \\
-14 \pm 13 \\
-22 \pm 12 \\
0.062\end{array}$ & $\begin{array}{l}1.1 \pm 0.1 \\
-18 \pm 11 \\
-13 \pm 13 \\
0.204\end{array}$ & $\begin{array}{l}\text { nd } \\
\text { nd } \\
\text { nd } \\
-\end{array}$ & $\begin{array}{l}0.14 \pm 0.03 \\
-25 \pm 12 \\
-9 \pm 15 \\
0.002\end{array}$ & $\begin{array}{l}1.4 \pm 0.1 \\
-54 \pm 10 \\
-51 \pm 9 \\
0.314\end{array}$ & $\begin{array}{l}1.4 \pm 0.1 \\
-62 \pm 39 \\
-59 \pm 43 \\
0.830\end{array}$ & $\begin{array}{l}4.1 \pm 0.1 \\
-39 \pm 13 \\
-35 \pm 14 \\
0.395\end{array}$ \\
\hline $\begin{array}{l}\text { Storage time } \\
\text { (ST) }\end{array}$ & $\begin{array}{l}12 \text { months } \\
18 \text { months } \\
p \text {-value } \\
(\mathrm{n}=18)\end{array}$ & $\begin{array}{l}-5 \pm 15 \\
-13 \pm 18 \\
0.164\end{array}$ & $\begin{array}{l}-26 \pm 14 \\
-40 \pm 14 \\
0.005\end{array}$ & $\begin{array}{l}-21 \pm 18 \\
-32 \pm 23 \\
0.143\end{array}$ & $\begin{array}{l}-1 \pm 19 \\
5 \pm 25 \\
0.463\end{array}$ & $\begin{array}{l}-14 \pm 11 \\
-22 \pm 14 \\
0.072\end{array}$ & $\begin{array}{l}-17 \pm 11 \\
-14 \pm 13 \\
0.449\end{array}$ & $\begin{array}{l}\text { nd } \\
\text { nd } \\
-\end{array}$ & $\begin{array}{l}-20 \pm 18 \\
-14 \pm 12 \\
0.314\end{array}$ & $\begin{array}{l}-53 \pm 10 \\
-52 \pm 9 \\
0.907\end{array}$ & $\begin{array}{l}-21 \pm 8 \\
-100^{\mathrm{a}} \\
<0.001\end{array}$ & $\begin{array}{l}-25 \pm 7 \\
-49 \pm 6 \\
<0.001\end{array}$ \\
\hline$(\mathrm{EB} \times \mathrm{ST})$ & $\begin{array}{l}p \text {-value } \\
(\mathrm{n}=36)\end{array}$ & 0.454 & 0.539 & 0.021 & 0.533 & 0.400 & 0.389 & - & 0.030 & 0.913 & 0.121 & 0.882 \\
\hline \multicolumn{13}{|c|}{ Melissa officinalis (lemon balm) } \\
\hline $\begin{array}{l}\text { Control (0 mont } \\
\text { Electron-beam } \\
\quad \text { (EB) }\end{array}$ & $\begin{array}{l}\text { hs, } 0 \text { kGy) } \\
0 \mathrm{kGy} \\
10 \mathrm{kGy} \\
p \text {-value } \\
(\mathrm{n}=18)\end{array}$ & $\begin{array}{l}1.2 \pm 0.1 \\
-9 \pm 13 \\
-9 \pm 16 \\
0.986\end{array}$ & $\begin{array}{l}1.0 \pm 0.1 \\
-49 \pm 9 \\
-17 \pm 17 \\
<0.001\end{array}$ & $\begin{array}{l}4.8 \pm 0.2 \\
-100^{\mathrm{a}} \\
-100^{\mathrm{a}} \\
-\end{array}$ & $\begin{array}{l}0.49 \pm 0.05 \\
-53 \pm 8 \\
7 \pm 20 \\
<0.001\end{array}$ & $\begin{array}{l}7.5 \pm 0.2 \\
-33 \pm 6 \\
-9 \pm 14 \\
<0.001\end{array}$ & $\begin{array}{l}0.5 \pm 0.1 \\
-25 \pm 14 \\
-5 \pm 22 \\
0.002\end{array}$ & $\begin{array}{l}0.26 \pm 0.04 \\
16 \pm 26 \\
-5 \pm 20 \\
0.010\end{array}$ & $\begin{array}{l}0.4 \pm 0.1 \\
14 \pm 25 \\
-5 \pm 19 \\
0.015\end{array}$ & $\begin{array}{l}4.1 \pm 0.2 \\
-32 \pm 12 \\
-36 \pm 10 \\
0.297\end{array}$ & $\begin{array}{l}\text { nd } \\
\text { nd } \\
\text { nd } \\
-\end{array}$ & $\begin{array}{l}5.3 \pm 0.3 \\
-21 \pm 11 \\
-21 \pm 10 \\
0.934\end{array}$ \\
\hline $\begin{array}{l}\text { Storage time } \\
\text { (ST) }\end{array}$ & $\begin{array}{l}12 \text { months } \\
18 \text { months } \\
p \text {-value } \\
(\mathrm{n}=18)\end{array}$ & $\begin{array}{l}-11 \pm 16 \\
-7 \pm 14 \\
0.429\end{array}$ & $\begin{array}{l}-32 \pm 20 \\
-34 \pm 23 \\
0.768\end{array}$ & $\begin{array}{l}-100^{\mathrm{a}} \\
-100^{\mathrm{a}} \\
-\end{array}$ & $\begin{array}{l}-27 \pm 29 \\
-20 \pm 39 \\
0.543\end{array}$ & $\begin{array}{l}-22 \pm 14 \\
-19 \pm 18 \\
0.588\end{array}$ & $\begin{array}{l}-17 \pm 17 \\
-13 \pm 24 \\
0.550\end{array}$ & $\begin{array}{l}-5 \pm 14 \\
15 \pm 30 \\
0.018\end{array}$ & $\begin{array}{l}10 \pm 23 \\
-1 \pm 24 \\
0.150\end{array}$ & $\begin{array}{l}-33 \pm 9 \\
-35 \pm 13 \\
0.572\end{array}$ & $\begin{array}{l}\text { nd } \\
\text { nd } \\
-\end{array}$ & $\begin{array}{l}-21 \pm 10 \\
-21 \pm 11 \\
0.813\end{array}$ \\
\hline$(\mathrm{EB} \times \mathrm{ST})$ & $\begin{array}{l}p \text {-value } \\
(\mathrm{n}=36)\end{array}$ & 0.487 & 0.423 & - & 0.046 & 0.179 & 0.271 & 0.600 & 0.732 & 0.385 & - & 0.807 \\
\hline \multicolumn{13}{|c|}{ Melittis melissophyllum (bastard balm) } \\
\hline $\begin{array}{l}\text { Control (0 mont } \\
\text { Electron-beam } \\
\quad \text { (EB) }\end{array}$ & $\begin{array}{l}\text { hs, } 0 \text { kGy) } \\
0 \mathrm{kGy} \\
10 \mathrm{kGy} \\
p \text {-value } \\
(\mathrm{n}=18)\end{array}$ & $\begin{array}{l}1.0 \pm 0.1 \\
-20 \pm 8 \\
-4 \pm 12 \\
<0.001\end{array}$ & $\begin{array}{l}0.8 \pm 0.1 \\
-25 \pm 7 \\
-5 \pm 7 \\
<0.001\end{array}$ & $\begin{array}{l}0.9 \pm 0.1 \\
-6 \pm 9 \\
-9 \pm 6 \\
0.326\end{array}$ & $\begin{array}{l}0.28 \pm 0.03 \\
504 \pm 218 \\
783 \pm 327 \\
0.005\end{array}$ & $\begin{array}{l}5.5 \pm 0.3 \\
11 \pm 10 \\
26 \pm 8 \\
<0.001\end{array}$ & $\begin{array}{l}1.4 \pm 0.1 \\
-15 \pm 8 \\
-15 \pm 8 \\
0.788\end{array}$ & $\begin{array}{l}0.17 \pm 0.01 \\
-20 \pm 12 \\
1 \pm 19 \\
<0.001\end{array}$ & $\begin{array}{l}6.0 \pm 0.3 \\
-63 \pm 2 \\
-72 \pm 5 \\
<0.001\end{array}$ & $\begin{array}{l}0.97 \pm 0.05 \\
-74 \pm 27 \\
-72 \pm 30 \\
0.809\end{array}$ & $\begin{array}{l}0.022 \pm 0.001 \\
-27 \pm 13 \\
-24 \pm 15 \\
0.569\end{array}$ & $\begin{array}{l}8.6 \pm 0.4 \\
-53 \pm 6 \\
-59 \pm 8 \\
0.010\end{array}$ \\
\hline $\begin{array}{l}\text { Storage time } \\
\text { (ST) }\end{array}$ & $\begin{array}{l}12 \text { months } \\
18 \text { months } \\
p \text {-value } \\
(\mathrm{n}=18)\end{array}$ & $\begin{array}{l}-11 \pm 12 \\
-13 \pm 14 \\
0.684\end{array}$ & $\begin{array}{l}-13 \pm 12 \\
-17 \pm 13 \\
0.284\end{array}$ & $\begin{array}{l}-5 \pm 7 \\
-9 \pm 7 \\
0.089\end{array}$ & $\begin{array}{l}748 \pm 339 \\
540 \pm 241 \\
0.041\end{array}$ & $\begin{array}{l}25 \pm 9 \\
12 \pm 11 \\
0.001\end{array}$ & $\begin{array}{l}-14 \pm 6 \\
-15 \pm 10 \\
0.746\end{array}$ & $\begin{array}{l}1 \pm 17 \\
-20 \pm 14 \\
<0.001\end{array}$ & $\begin{array}{l}-65 \pm 3 \\
-70 \pm 7 \\
0.006\end{array}$ & $\begin{array}{l}-45 \pm 9 \\
-100^{\mathrm{a}} \\
<0.001\end{array}$ & $\begin{array}{l}-29 \pm 14 \\
-22 \pm 15 \\
0.137\end{array}$ & $\begin{array}{l}-50 \pm 3 \\
-63 \pm 5 \\
<0.001\end{array}$ \\
\hline$(\mathrm{EB} \times \mathrm{ST})$ & $\begin{array}{l}p \text {-value } \\
(\mathrm{n}=36)\end{array}$ & 0.671 & 0.446 & 0.065 & 0.727 & 0.688 & 0.183 & 0.078 & $<0.001$ & 0.296 & 0.321 & 0.075 \\
\hline \multicolumn{13}{|c|}{ Mentha piperita (peppermint) } \\
\hline $\begin{array}{l}\text { Control (0 mont } \\
\text { Electron-beam } \\
\quad(\mathrm{EB})\end{array}$ & $\begin{array}{l}\text { hs, } 0 \text { kGy) } \\
0 \mathrm{kGy} \\
10 \mathrm{kGy} \\
p \text {-value } \\
(\mathrm{n}=18)\end{array}$ & $\begin{array}{l}0.47 \pm 0.05 \\
-13 \pm 14 \\
3 \pm 20 \\
0.012\end{array}$ & $\begin{array}{l}0.30 \pm 0.05 \\
-91 \pm 2 \\
-88 \pm 3 \\
<0.001\end{array}$ & $\begin{array}{l}0.7 \pm 0.1 \\
-45 \pm 28 \\
4 \pm 62 \\
0.005\end{array}$ & $\begin{array}{l}1.0 \pm 0.1 \\
-33 \pm 20 \\
15 \pm 50 \\
0.001\end{array}$ & $\begin{array}{l}2.4 \pm 0.2 \\
-75 \pm 4 \\
-67 \pm 5 \\
<0.001\end{array}$ & $\begin{array}{l}1.1 \pm 0.1 \\
-26 \pm 19 \\
-16 \pm 13 \\
0.070\end{array}$ & $\begin{array}{l}0.040 \pm 0.003 \\
-19 \pm 25 \\
-17 \pm 13 \\
0.723\end{array}$ & $\begin{array}{l}0.9 \pm 0.1 \\
-25 \pm 8 \\
-39 \pm 8 \\
<0.001\end{array}$ & $\begin{array}{l}\text { nd } \\
\text { nd } \\
\text { nd } \\
-\end{array}$ & $\begin{array}{l}8.5 \pm 0.2 \\
-18 \pm 12 \\
-34 \pm 5 \\
<0.001\end{array}$ & $\begin{array}{l}10.6 \pm 0.3 \\
-21 \pm 8 \\
-34 \pm 3 \\
<0.001\end{array}$ \\
\hline $\begin{array}{l}\text { Storage time } \\
\text { (ST) }\end{array}$ & $\begin{array}{l}12 \text { months } \\
18 \text { months } \\
p \text {-value } \\
(\mathrm{n}=18)\end{array}$ & $\begin{array}{l}-2 \pm 17 \\
-7 \pm 21 \\
0.416\end{array}$ & $\begin{array}{l}-87 \pm 2 \\
-92 \pm 2 \\
<0.001\end{array}$ & $\begin{array}{l}-5 \pm 60 \\
-36 \pm 42 \\
0.079\end{array}$ & $\begin{array}{l}-1 \pm 49 \\
-17 \pm 40 \\
0.299\end{array}$ & $\begin{array}{l}-68 \pm 5 \\
-74 \pm 6 \\
0.002\end{array}$ & $\begin{array}{l}-22 \pm 17 \\
-20 \pm 17 \\
0.740\end{array}$ & $\begin{array}{l}-29 \pm 9 \\
-6 \pm 20 \\
<0.001\end{array}$ & $\begin{array}{l}-29 \pm 10 \\
-35 \pm 11 \\
0.133\end{array}$ & $\begin{array}{l}\text { nd } \\
\text { nd } \\
-\end{array}$ & $\begin{array}{l}-28 \pm 13 \\
-24 \pm 11 \\
0.423\end{array}$ & $\begin{array}{l}-28 \pm 9 \\
-27 \pm 9 \\
0.677\end{array}$ \\
\hline$(\mathrm{EB} \times \mathrm{ST})$ & $\begin{array}{l}p \text {-value } \\
(\mathrm{n}=36)\end{array}$ & 0.640 & 0.070 & 0.841 & 0.618 & 0.953 & 0.788 & 0.032 & 0.234 & - & 0.132 & 0.258 \\
\hline
\end{tabular}

\footnotetext{
a This parameter was not detected in the samples treated under these conditions. nd - not detected.
} 
selected dimensions was chosen according to the eigenvalue, the Cronbach's alpha and the percentage of total explained variance. In all cases, the plotted dimensions were kept to a maximum of three to allow their graphical interpretation in a meaningful way.

\section{Results and discussion}

In previous studies conducted with the plant species assayed herein, the effects of e-beam (EB) irradiation were evaluated promptly (as soon as possible) after irradiation treatment (Pereira, Antonio, Rafalski, et al., 2015). Now, the main purpose is performing a comprehensive characterization of the effects of e-beam irradiation throughout storage time (ST). To evaluate the effects of EB independently of ST and vice versa, the results of each factor were consecutively aggregated. Therefore, the variation in the values obtained for each parameter should not be regarded as a typical standard deviation, but instead as the amplitude of values obtained in samples submitted to different EB doses or ST. To avoid the variability induced by plant species (some evaluated parameters presented highly different magnitudes among species), the results were evaluated considering percentage variations in comparison to the respective control (unirradiated fresh samples). Every time the variation laid below 5\%, either representing an increase or a decrease, it was assumed that the irradiation effect was not significant.

\subsection{Effects on chemical parameters}

Concerning proximate composition and colour parameters (Table 1 ), the interaction $(\mathrm{EB} \times \mathrm{ST})$ among factor was significant $(p<0.05)$ only in few occasions, thereby allowing performing multiple comparisons in most situations. Regarding nutritional parameters, fat (especially in A. citrodora and M. piperita) and ash content tended to decrease along ST, and this reduction could not be attenuated by EB. Among colour parameters, the only significant differences were found in redness $\left(a^{*}\right)$, which decreased along ST in all plant species.

Nevertheless, the main result observable in Table 1 is the general absence of a significant effect of both factors in the assayed parameters. In fact, in $62.5 \%$ of the cases ( 40 out of 64 ) the percentage variation in comparison to the corresponding control laid below $5 \%$, indicating and apparent stability of the nutritional (except fat and ash) and colour parameters (except $a^{*}$ ), which might be considered as an indicator of the suitability of EB as a conservation methodology for these plants. The maintenance of $L^{*}$ and $b^{*}$, in particular, in association with the decrease in $a^{*}$ (which indicate "greener" samples) is a positive result, considering that colour parameters represent a good measure of adequate post-harvest preservation processes (Hsu, Simonne, Jitareerat, \& Marshall, 2010; Jo, Son, Shin, \& Byun, 2003).

Concerning the effects over hydrophilic compounds (Table 2), namely free sugars and organic acids, there was an evident

Table 3

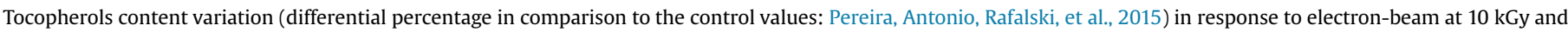
storage time. The results are presented as mean \pm standard deviation.

\begin{tabular}{|c|c|c|c|c|c|}
\hline & & $\alpha$-Tocopherol & $\beta$-Tocopherol & $\gamma$-Tocopherol & Total tocopherols \\
\hline $\begin{array}{l}\text { Aloysia citrodora (lemon ve } \\
\text { Control ( } 0 \text { months, } 0 \mathrm{kGy} \text { ) } \\
\text { Electron-beam (EB) }\end{array}$ & $\begin{array}{l}0 \mathrm{kGy} \\
10 \mathrm{kGy} \\
p \text {-value }(\mathrm{n}=18)\end{array}$ & $\begin{array}{l}15.3 \pm 0.4 \\
-77 \pm 5 \\
-74 \pm 7 \\
0.148\end{array}$ & $\begin{array}{l}0.41 \pm 0.04 \\
-31 \pm 14 \\
-11 \pm 21 \\
0.003\end{array}$ & $\begin{array}{l}1.8 \pm 0.1 \\
-81 \pm 5 \\
-78 \pm 7 \\
0.236\end{array}$ & $\begin{array}{l}17.5 \pm 0.4 \\
-77 \pm 5 \\
-74 \pm 6 \\
0.113\end{array}$ \\
\hline $\begin{array}{l}\text { Storage time }(\mathrm{ST}) \\
(\mathrm{EB} \times \mathrm{ST})\end{array}$ & $\begin{array}{l}12 \text { months } \\
18 \text { months } \\
p \text {-value }(\mathrm{n}=18) \\
p \text {-value }(\mathrm{n}=36)\end{array}$ & $\begin{array}{l}-70 \pm 4 \\
-81 \pm 2 \\
<0.001 \\
0.033\end{array}$ & $\begin{array}{l}-21 \pm 16 \\
-21 \pm 24 \\
0.990 \\
0.363\end{array}$ & $\begin{array}{l}-75 \pm 4 \\
-84 \pm 3 \\
<0.001 \\
0.138\end{array}$ & $\begin{array}{l}-70 \pm 3 \\
-81 \pm 2 \\
<0.001 \\
0.010\end{array}$ \\
\hline $\begin{array}{l}\text { Melissa officinalis (lemon ba } \\
\text { Control ( } 0 \text { months, } 0 \mathrm{kGy} \text { ) } \\
\text { Electron-beam (EB) }\end{array}$ & $\begin{array}{l}0 \mathrm{kGy} \\
10 \mathrm{kGy} \\
p \text {-value }(\mathrm{n}=18)\end{array}$ & $\begin{array}{l}29 \pm 1 \\
-75 \pm 2 \\
-69 \pm 4 \\
<0.001\end{array}$ & $\begin{array}{l}1.3 \pm 0.1 \\
-63 \pm 10 \\
-52 \pm 22 \\
0.054\end{array}$ & $\begin{array}{l}1.5 \pm 0.1 \\
-43 \pm 14 \\
-31 \pm 18 \\
0.037\end{array}$ & $\begin{array}{l}32 \pm 1 \\
-73 \pm 2 \\
-66 \pm 5 \\
<0.001\end{array}$ \\
\hline $\begin{array}{l}\text { Storage time }(\mathrm{ST}) \\
(\mathrm{EB} \times \mathrm{ST})\end{array}$ & $\begin{array}{l}12 \text { months } \\
18 \text { months } \\
p \text {-value }(\mathrm{n}=18) \\
p \text {-value }(\mathrm{n}=36)\end{array}$ & $\begin{array}{l}-70 \pm 5 \\
-74 \pm 2 \\
0.002 \\
<0.001\end{array}$ & $\begin{array}{l}-45 \pm 16 \\
-70 \pm 7 \\
<0.001 \\
0.002\end{array}$ & $\begin{array}{l}-25 \pm 13 \\
-49 \pm 11 \\
<0.001 \\
0.437\end{array}$ & $\begin{array}{l}-67 \pm 5 \\
-73 \pm 3 \\
<0.001 \\
<0.001\end{array}$ \\
\hline \multicolumn{6}{|c|}{ Melittis melissophyllum (bastard balm) } \\
\hline $\begin{array}{l}\text { Control ( } 0 \text { months, } 0 \mathrm{kGy} \text { ) } \\
\text { Electron-beam (EB) }\end{array}$ & $\begin{array}{l}0 \mathrm{kGy} \\
10 \mathrm{kGy} \\
p \text {-value }(\mathrm{n}=18)\end{array}$ & $\begin{array}{l}0.88 \pm 0.05 \\
-71 \pm 8 \\
-80 \pm 4 \\
<0.001\end{array}$ & $\begin{array}{l}13.4 \pm 0.3 \\
-100^{\mathrm{a}} \\
-100^{\mathrm{a}} \\
-\end{array}$ & $\begin{array}{l}0.18 \pm 0.02 \\
-9 \pm 11 \\
-20 \pm 5 \\
0.220\end{array}$ & $\begin{array}{l}14.6 \pm 0.4 \\
-16 \pm 10 \\
-20 \pm 9 \\
0.223\end{array}$ \\
\hline Storage time (ST) & $\begin{array}{l}12 \text { months } \\
18 \text { months } \\
p \text {-value }(\mathrm{n}=18)\end{array}$ & $\begin{array}{l}-72 \pm 8 \\
-78 \pm 6 \\
0.018\end{array}$ & $\begin{array}{l}-100^{\mathrm{a}} \\
-100^{\mathrm{a}} \\
-\end{array}$ & $\begin{array}{l}-5 \pm 9 \\
-17 \pm 7 \\
<0.001\end{array}$ & $\begin{array}{l}-12 \pm 8 \\
-24 \pm 7 \\
<0.001\end{array}$ \\
\hline $\begin{array}{l}(\mathrm{EB} \times \mathrm{ST}) \\
\text { Mentha piperita (peppermir }\end{array}$ & $p$-value $(\mathrm{n}=36)$ & 0.131 & - & 0.234 & 0.653 \\
\hline $\begin{array}{l}\text { Control ( } 0 \text { months, } 0 \mathrm{kGy} \text { ) } \\
\text { Electron-beam (EB) }\end{array}$ & $\begin{array}{l}0 \mathrm{kGy} \\
10 \mathrm{kGy} \\
p \text {-value }(\mathrm{n}=18)\end{array}$ & $\begin{array}{l}16.5 \pm 0.4 \\
-64 \pm 8 \\
-64 \pm 10 \\
0.915\end{array}$ & $\begin{array}{l}1.1 \pm 0.1 \\
-100^{\mathrm{a}} \\
-100^{\mathrm{a}} \\
-\end{array}$ & $\begin{array}{l}1.8 \pm 0.1 \\
-63 \pm 11 \\
-52 \pm 13 \\
0.011\end{array}$ & $\begin{array}{l}19.7 \pm 0.5 \\
-65 \pm 8 \\
-65 \pm 10 \\
0.828\end{array}$ \\
\hline Storage time (ST) & $\begin{array}{l}12 \text { months } \\
18 \text { months } \\
p \text {-value }(\mathrm{n}=18) \\
p \text {-value }(\mathrm{n}=36)\end{array}$ & $\begin{array}{l}-56 \pm 3 \\
-73 \pm 2 \\
<0.001 \\
0.004\end{array}$ & $\begin{array}{l}-100^{\mathrm{a}} \\
-100^{\mathrm{a}} \\
- \\
-\end{array}$ & $\begin{array}{l}-48 \pm 9 \\
-68 \pm 7 \\
<0.001 \\
0.973\end{array}$ & $\begin{array}{l}-57 \pm 3 \\
-73 \pm 2 \\
<0.001 \\
0.005\end{array}$ \\
\hline
\end{tabular}

\footnotetext{
a This parameter was not detected in the samples treated under these conditions.
} 
Table 4

Major fatty acids variation (differential percentage in comparison to the control values: Pereira, Antonio, Rafalski, et al., 2015) in response to electron-beam at $10 \mathrm{kGy}$ and storage time. The results are presented as mean \pm standard deviation.

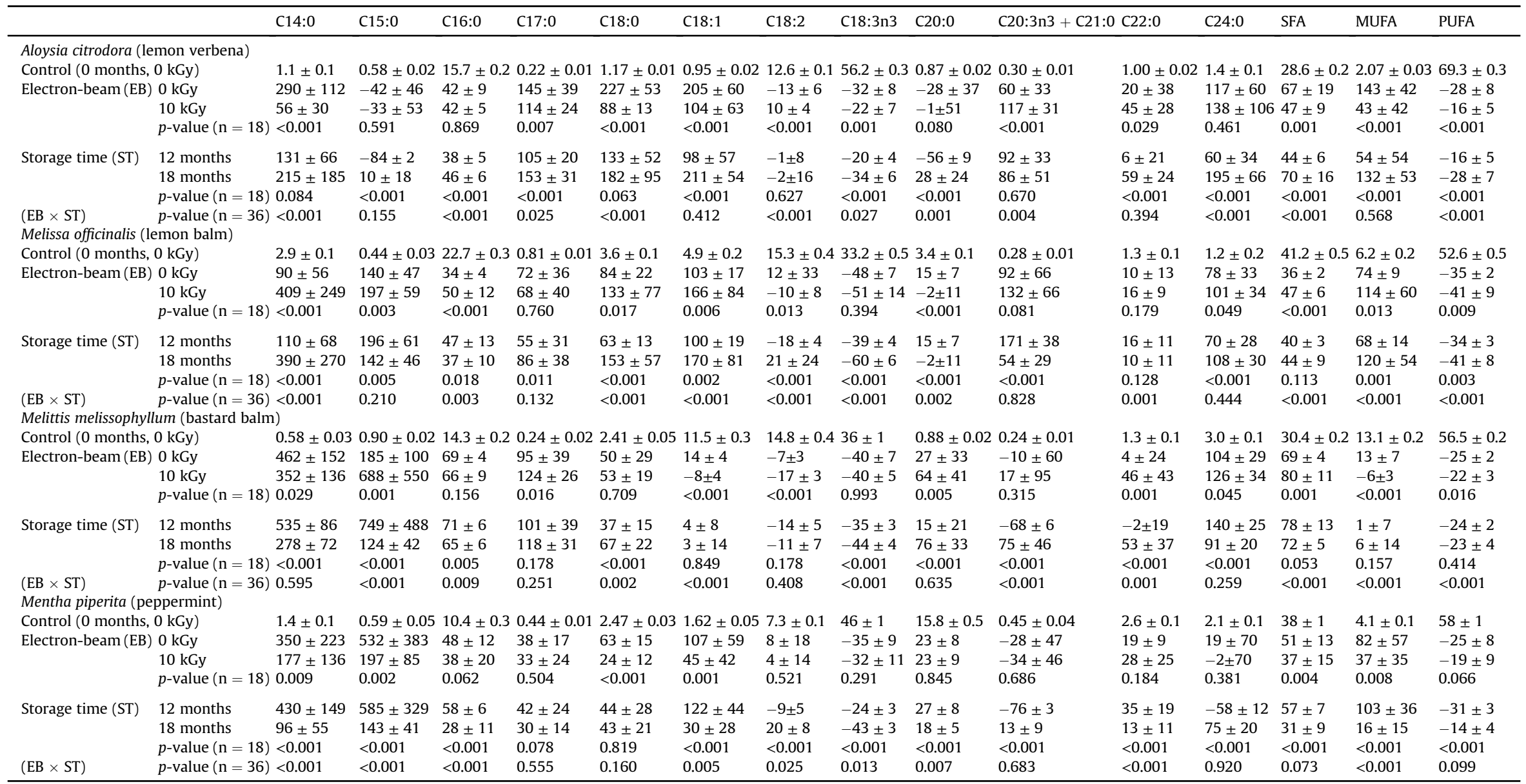


tendency to their decrease throughout ST, except for trehalose in M. melissophyllum and quinic acid and malic acid in M. officinalis. It is also noticeable that the reducing effect induced by ST was more pronounced (except for $A$. citrodora) than the one resulting from EB. Furthermore, the decrease observed after 18 months was significantly stronger for glucose (in A. citrodora and M. piperita), sucrose, trehalose and total sugars (all in M. piperita), quinic and shikimic acid (in M. melissophyllum), citric acid (in A. citrodora) and total organic acids (in A. citrodora and M. melissophyllum). However, the overall reduction of free sugars and organic acids was attenuated by EB treatment in several occurrences, namely in fructose (in M. melissophyllum and M. piperita), glucose (in all species except A. citrodora), sucrose (in M. piperita), trehalose and total sugars (in all species except $A$. citrodora) and malic acid (in $A$. citrodora). In general, the compounds that presented the highest differences were glucose, sucrose, shikimic and citric acids.

The contents in tocopherols showed also a marked decrease throughout ST, which was significantly higher (except for $\beta$ tocopherol) after 18 months, when compared to the results measured after 12 months, (Table 3). The applied EB treatment could not prevent those significant reductions in most occasions, but it has a positive effect in $\alpha$-tocopherol (in $M$. officinalis), $\beta$ tocopherol (in A. citrodora), $\gamma$-tocopherol (in M. officinalis and $M$. piperita) and total tocopherols (in $M$. officinalis). Except for A. citrodora, $\alpha$ and $\beta$ isoforms seemed to be the most susceptible to EB irradiation, in line with previously reported results (Warner Miller, \& Demurin, 2008).

A large number of fatty acids, namely C6:0, C8:0, C10:0, C11:0 (except in M. melissophyllum), C12:0, C13:0, C14:1 (except in A. citrodora and M. melissophyllum), C15:1, C16:1, C18:3n6 (only in M. melissophyllum), C20:1, C20:2, C20:5n3 (except in A. citrodora and $M$. melissophyllum), C22:1 (except in $M$. officinalis and M. melissophyllum), C22:6n3 (except in $M$. officinalis and A. citrodora) and (C23:0.) were detected in relative percentages lower than $1 \%$. Accordingly, and despite the variations in those minor fatty acids were also included in the PCA, they were not included in Table 4.

Contrary to previous parameters, the interaction among factors was significant in most cases. By restricting the analysis to those results were the interaction was not significant and at least one of the factors had a significant effect per se, some defined tendencies were noticeable. While non-irradiated samples of $A$. citrodora and

Table 5

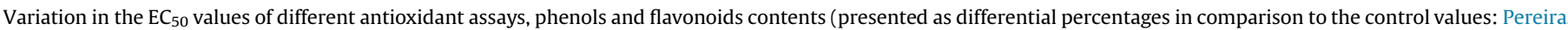
Antonio, Rafalski, et al., 2015) in response to electron-beam at $10 \mathrm{kGy}$ and storage time. The results are presented as mean \pm standard deviation.

\begin{tabular}{|c|c|c|c|c|c|c|c|c|c|c|c|c|c|}
\hline & & \multicolumn{2}{|c|}{$\begin{array}{l}\text { DPPH scavenging } \\
\text { activity }\end{array}$} & \multicolumn{2}{|l|}{$\begin{array}{l}\text { Reducing } \\
\text { power }\end{array}$} & \multicolumn{2}{|c|}{$\begin{array}{l}\beta \text {-carotene bleaching } \\
\text { inhibition }\end{array}$} & \multicolumn{2}{|c|}{$\begin{array}{l}\text { TBARS formation } \\
\text { inhibition }\end{array}$} & \multicolumn{2}{|l|}{ Phenols } & \multicolumn{2}{|l|}{ Flavonoids } \\
\hline & & Infusion & $\mathrm{leOH}$ & on & $\mathrm{MeOH}$ & on & $\mathrm{MeOH}$ & fusion & $\mathrm{MeOH}$ & Infusion & $\mathrm{MeOH}$ & Infusion & $\mathrm{MeOH}$ \\
\hline \multicolumn{14}{|c|}{ Aloysia citrodora (lemon verbena) } \\
\hline $\begin{array}{l}\text { Control } \\
\text { (0 months) }\end{array}$ & 0 kGy & $232 \pm 8$ & $39 \pm 4$ & $169 \pm 1$ & $22.8 \pm 0.3$ & $580 \pm 31$ & $208 \pm 9$ & $121 \pm 5$ & $24 \pm 4$ & $134 \pm 8$ & $665 \pm 13$ & $92 \pm 1$ & $369 \pm 5$ \\
\hline Electron-beam (EB) & $\begin{array}{l}0 \mathrm{kGy} \\
10 \mathrm{kGy} \\
p \text {-value }(\mathrm{n}=18)\end{array}$ & $\begin{array}{l}-9 \pm 12 \\
5 \pm 16 \\
0.008\end{array}$ & $\begin{array}{l}-4 \pm 14 \\
5 \pm 12 \\
0.035\end{array}$ & $\begin{array}{l}-6 \pm 14 \\
9 \pm 10 \\
0.001\end{array}$ & $\begin{array}{l}-10 \pm 2 \\
-1 \pm 1 \\
<0.001\end{array}$ & $\begin{array}{l}-74 \pm 12 \\
-88 \pm 3 \\
<0.001\end{array}$ & $\begin{array}{l}-85 \pm 1 \\
-91 \pm 2 \\
<0.001\end{array}$ & $\begin{array}{l}181 \pm 78 \\
422 \pm 19 \\
<0.001\end{array}$ & $\begin{array}{l}4 \pm 55 \\
70 \pm 49 \\
0.001\end{array}$ & $\begin{array}{l}12 \pm 8 \\
-2 \pm 33 \\
0.098\end{array}$ & $\begin{array}{l}10 \pm 7 \\
2 \pm 8 \\
0.003\end{array}$ & $\begin{array}{l}-23 \pm 32 \\
-24 \pm 40 \\
0.955\end{array}$ & $\begin{array}{l}16 \pm 4 \\
5 \pm 3 \\
<0.001\end{array}$ \\
\hline Storage time (ST) & $\begin{array}{l}12 \text { months } \\
18 \text { months } \\
p \text {-value }(\mathrm{n}=18) \\
p \text {-value }(\mathrm{n}=36)\end{array}$ & $\begin{array}{l}-4 \pm 7 \\
-1 \pm 21 \\
0.468 \\
<0.001\end{array}$ & $\begin{array}{l}-11 \pm 7 \\
12 \pm 6 \\
<0.001 \\
0.198\end{array}$ & $\begin{array}{l}3 \pm 4 \\
-1 \pm 20 \\
0.424 \\
<0.001\end{array}$ & $\begin{array}{l}-6 \pm 6 \\
-4 \pm 3 \\
0.383 \\
<0.001\end{array}$ & $\begin{array}{l}-74 \pm 13 \\
-87 \pm 3 \\
<0.001 \\
<0.001\end{array}$ & $\begin{array}{l}-89 \pm 4 \\
-87 \pm 3 \\
0.111 \\
0.002\end{array}$ & $\begin{array}{l}339 \pm 93 \\
264 \pm 160 \\
0.093 \\
<0.001\end{array}$ & $\begin{array}{l}-7 \pm 35 \\
81 \pm 50 \\
<0.001 \\
0.974\end{array}$ & $\begin{array}{l}20 \pm 13 \\
-10 \pm 24 \\
<0.001 \\
<0.001\end{array}$ & $\begin{array}{l}13 \pm 5 \\
-1 \pm 5 \\
<0.001 \\
0.268\end{array}$ & $\begin{array}{l}11 \pm 9 \\
-58 \pm 5 \\
<0.001 \\
<0.001\end{array}$ & $\begin{array}{l}13 \pm 7 \\
7 \pm 5 \\
0.003 \\
0.008\end{array}$ \\
\hline \multicolumn{14}{|c|}{ Melissa officinalis (lemon balm) } \\
\hline $\begin{array}{l}\text { Control } \\
\text { (0 months) }\end{array}$ & 0 kGy & $101 \pm 3$ & $67 \pm 1$ & $80 \pm 1$ & $44 \pm 1$ & $165 \pm 4$ & $125 \pm 3$ & $145 \pm 8$ & $31.5 \pm 0.3$ & $100 \pm 1$ & $829 \pm 6$ & $63 \pm 1$ & $448 \pm 4$ \\
\hline Electron-beam (EB) & $\begin{array}{l}0 \mathrm{kGy} \\
10 \mathrm{kGy} \\
p \text {-value }(\mathrm{n}=18)\end{array}$ & $\begin{array}{l}12 \pm 10 \\
28 \pm 8 \\
<0.001\end{array}$ & $\begin{array}{l}-16 \pm 14 \\
-13 \pm 13 \\
0.588\end{array}$ & $\begin{array}{l}15 \pm 14 \\
13 \pm 5 \\
0.697\end{array}$ & $\begin{array}{l}-22 \pm 1 \\
-29 \pm 3 \\
<0.001\end{array}$ & $\begin{array}{l}4 \pm 40 \\
-61 \pm 8 \\
<0.001\end{array}$ & $\begin{array}{l}-47 \pm 22 \\
-44 \pm 22 \\
0.713\end{array}$ & $\begin{array}{l}20 \pm 25 \\
43 \pm 18 \\
0.003\end{array}$ & $\begin{array}{l}-1 \pm 5 \\
43 \pm 31 \\
<0.001\end{array}$ & $\begin{array}{l}-31 \pm 29 \\
-26 \pm 33 \\
0.668\end{array}$ & $\begin{array}{l}1 \pm 28 \\
9 \pm 33 \\
0.430\end{array}$ & $\begin{array}{l}-29 \pm 14 \\
-33 \pm 12 \\
0.326\end{array}$ & $\begin{array}{l}38 \pm 4 \\
44 \pm 7 \\
0.005\end{array}$ \\
\hline Storage time (ST) & $\begin{array}{l}12 \text { months } \\
18 \text { months } \\
p \text {-value }(\mathrm{n}=18)\end{array}$ & $\begin{array}{l}14 \pm 10 \\
25 \pm 12 \\
0.006\end{array}$ & $\begin{array}{l}-25 \pm 7 \\
-4 \pm 10 \\
<0.001\end{array}$ & $\begin{array}{l}23 \pm 6 \\
5 \pm 4 \\
<0.001\end{array}$ & $\begin{array}{l}-27 \pm 5 \\
-24 \pm 2 \\
0.076\end{array}$ & $\begin{array}{l}-43 \pm 35 \\
-15 \pm 47 \\
0.048\end{array}$ & $\begin{array}{l}-25 \pm 5 \\
-66 \pm 7 \\
<0.001\end{array}$ & $\begin{array}{l}49 \pm 11 \\
15 \pm 22 \\
<0.001\end{array}$ & $\begin{array}{l}31 \pm 40 \\
12 \pm 14 \\
0.081\end{array}$ & $\begin{array}{l}1 \pm 6 \\
-59 \pm 1 \\
<0.001\end{array}$ & $\begin{array}{l}34 \pm 7 \\
-26 \pm 2 \\
<0.001\end{array}$ & $\begin{array}{l}-20 \pm 8 \\
-42 \pm 6 \\
<0.001\end{array}$ & $\begin{array}{l}45 \pm 6 \\
37 \pm 2 \\
<0.001\end{array}$ \\
\hline $\begin{array}{l}(\mathrm{EB} \times \mathrm{ST}) \\
\text { Melittis melissophyll }\end{array}$ & $\begin{array}{l}p \text {-value }(\mathrm{n}=36) \\
\text { um (bastard balm) }\end{array}$ & 0.309 & 0.812 & $<0.001$ & $<0.001$ & 0.019 & 0.741 & 0.002 & $<0.001$ & 0.002 & $<0.001$ & 0.793 & $<0.001$ \\
\hline $\begin{array}{l}\text { Control } \\
\text { (0 months) }\end{array}$ & 0 kGy & $583 \pm 24$ & $354 \pm 39$ & $512 \pm 16$ & $249 \pm 2$ & $1648 \pm 154$ & $447 \pm 66$ & $589 \pm 25$ & $187 \pm 9$ & $70 \pm 4$ & $160 \pm 3$ & $29 \pm 2$ & $108 \pm 4$ \\
\hline Electron-beam (EB & $\begin{array}{l}0 \mathrm{kGy} \\
10 \mathrm{kGy} \\
p \text {-value }(\mathrm{n}=18)\end{array}$ & $\begin{array}{l}-51 \pm 32 \\
-54 \pm 29 \\
0.743\end{array}$ & $\begin{array}{l}-60 \pm 17 \\
-38 \pm 29 \\
0.013\end{array}$ & $\begin{array}{l}-35 \pm 42 \\
-38 \pm 40 \\
0.824\end{array}$ & $\begin{array}{l}-42 \pm 34 \\
11 \pm 70 \\
0.007\end{array}$ & $\begin{array}{l}-95 \pm 2 \\
-93 \pm 3 \\
0.022\end{array}$ & $\begin{array}{l}-82 \pm 7 \\
-74 \pm 14 \\
0.035\end{array}$ & $\begin{array}{l}-18 \pm 50 \\
-32 \pm 32 \\
0.330\end{array}$ & $\begin{array}{l}-77 \pm 11 \\
-68 \pm 12 \\
0.036\end{array}$ & $\begin{array}{l}56 \pm 44 \\
66 \pm 30 \\
0.413\end{array}$ & $\begin{array}{l}110 \pm 88 \\
104 \pm 96 \\
0.858\end{array}$ & $\begin{array}{l}82 \pm 59 \\
98 \pm 55 \\
0.398\end{array}$ & $\begin{array}{l}159 \pm 143 \\
107 \pm 122 \\
0.243\end{array}$ \\
\hline Storage time (ST) & $\begin{array}{l}12 \text { months } \\
18 \text { months } \\
p \text {-value }(\mathrm{n}=18)\end{array}$ & $\begin{array}{l}-23 \pm 7 \\
-82 \pm 1 \\
<0.001\end{array}$ & $\begin{array}{l}-27 \pm 17 \\
-72 \pm 5 \\
<0.001\end{array}$ & $\begin{array}{l}4 \pm 2 \\
-76 \pm 1 \\
<0.001\end{array}$ & $\begin{array}{l}35 \pm 45 \\
-66 \pm 10 \\
<0.001\end{array}$ & $\begin{array}{l}-91 \pm 2 \\
-96 \pm 1 \\
<0.001\end{array}$ & $\begin{array}{l}-68 \pm 7 \\
-88 \pm 2 \\
<0.001\end{array}$ & $\begin{array}{l}15 \pm 18 \\
-64 \pm 3 \\
<0.001\end{array}$ & $\begin{array}{l}-62 \pm 5 \\
-83 \pm 4 \\
<0.001\end{array}$ & $\begin{array}{l}26 \pm 17 \\
97 \pm 3 \\
<0.001\end{array}$ & $\begin{array}{l}18 \pm 7 \\
196 \pm 10 \\
<0.001\end{array}$ & $\begin{array}{l}35 \pm 11 \\
146 \pm 7 \\
<0.001\end{array}$ & $\begin{array}{l}4 \pm 17 \\
261 \pm 38 \\
<0.001\end{array}$ \\
\hline $\begin{array}{l}(\mathrm{EB} \times \mathrm{ST}) \\
\text { Mentha piperita }(\mathrm{pe}\end{array}$ & $\begin{array}{l}p \text {-value }(\mathrm{n}=36) \\
\text { ppermint })\end{array}$ & 0.050 & $<0.001$ & $<0.001$ & $<0.001$ & $<0.001$ & $<0.001$ & $<0.001$ & 0.482 & $<0.001$ & 0.001 & $<0.001$ & $<0.001$ \\
\hline $\begin{array}{l}\text { Control } \\
\text { ( } 0 \text { months) }\end{array}$ & 0 kGy & $184 \pm 5$ & $83 \pm 7$ & $119 \pm 2$ & $52 \pm 2$ & $597 \pm 44$ & $184 \pm 5$ & $54 \pm 6$ & $11 \pm 1$ & $218 \pm 2$ & $591 \pm 19$ & $117 \pm 2$ & $319 \pm 6$ \\
\hline Electron-beam (EB & $\begin{array}{l}0 \mathrm{kGy} \\
10 \mathrm{kGy} \\
p \text {-value }(\mathrm{n}=18)\end{array}$ & $\begin{array}{l}143 \pm 11 \\
183 \pm 17 \\
<0.001\end{array}$ & $\begin{array}{l}127 \pm 31 \\
158 \pm 35 \\
0.009\end{array}$ & $\begin{array}{l}18 \pm 2 \\
-12 \pm 2 \\
<0.001\end{array}$ & $\begin{array}{l}1 \pm 1 \\
-7 \pm 6 \\
<0.001\end{array}$ & $\begin{array}{l}-73 \pm 10 \\
-79 \pm 10 \\
0.093\end{array}$ & $\begin{array}{l}-13 \pm 33 \\
-54 \pm 4 \\
<0.001\end{array}$ & $\begin{array}{l}79 \pm 10 \\
50 \pm 30 \\
0.001\end{array}$ & $\begin{array}{l}125 \pm 37 \\
-29 \pm 8 \\
<0.001\end{array}$ & $\begin{array}{l}-29 \pm 24 \\
-32 \pm 23 \\
0.728\end{array}$ & $\begin{array}{l}7 \pm 5 \\
20 \pm 15 \\
0.003\end{array}$ & $\begin{array}{l}-23 \pm 33 \\
-20 \pm 36 \\
0.770\end{array}$ & $\begin{array}{l}6 \pm 7 \\
21 \pm 15 \\
0.001\end{array}$ \\
\hline Storage time (ST) & $\begin{array}{l}12 \text { months } \\
18 \text { months } \\
p \text {-value }(\mathrm{n}=18) \\
p \text {-value }(\mathrm{n}=36)\end{array}$ & $\begin{array}{l}160 \pm 24 \\
165 \pm 26 \\
0.531 \\
0.588\end{array}$ & $\begin{array}{l}111 \pm 16 \\
173 \pm 19 \\
<0.001 \\
0.190\end{array}$ & $\begin{array}{l}5 \pm 15 \\
1 \pm 16 \\
0.514 \\
0.047\end{array}$ & $\begin{array}{l}-6 \pm 7 \\
-1 \pm 1 \\
0.006 \\
<0.001\end{array}$ & $\begin{array}{l}-66 \pm 4 \\
-85 \pm 4 \\
<0.001 \\
0.872\end{array}$ & $\begin{array}{l}-16 \pm 37 \\
-51 \pm 7 \\
0.001 \\
<0.001\end{array}$ & $\begin{array}{l}53 \pm 33 \\
76 \pm 10 \\
0.014 \\
<0.001\end{array}$ & $\begin{array}{l}55 \pm 87 \\
41 \pm 79 \\
0.634 \\
0.297\end{array}$ & $\begin{array}{l}-8 \pm 2 \\
-53 \pm 1 \\
<0.001 \\
<0.001\end{array}$ & $\begin{array}{l}23 \pm 12 \\
4 \pm 2 \\
<0.001 \\
<0.001\end{array}$ & $\begin{array}{l}12 \pm 3 \\
-55 \pm 1 \\
<0.001 \\
<0.001\end{array}$ & $\begin{array}{l}24 \pm 12 \\
3 \pm 3 \\
<0.001 \\
<0.001\end{array}$ \\
\hline
\end{tabular}


M. piperita presented the highest increase in saturated fatty acids, the same effect was observed in the irradiated samples of M. officinalis and M. melissophyllum. On the other hand, monounsaturated fatty acids (MUFA) suffer, in general, higher increase in irradiated samples, while the reduction in polyunsaturated fatty acids (PUFA), such as C18:2n6, C18:3n3, was similar, independently of EB, ST and plant species. Nevertheless, EB treatment attenuated PUFA content in all plant species, except $M$. piperita.

\subsection{Effects on antioxidant parameters}

In line with the verified factors for the fatty acids variation, the interaction among factors was significant $(p<0.05)$ in several occasions, thereby restricting multiple comparisons to specific cases (in reducing power, in particular, no comparisons could be made). In general, changes in antioxidant activity were influenced by extract type (infusion or methanolic) and plant species (Table 5). The ability to scavenge DPPH radicals lowered in M. officinalis (infusion) and $M$. piperita, in both extracts (despite the protective effect exerted by EB treatment), as evidenced by the positive variation of their $\mathrm{EC}_{50}$ values; on the other hand, DPPH scavenging activity increased in the methanolic extracts of $M$. officinalis and in M. melissophyllum infusions. Regarding the capacity to inhibit $\beta$ carotene bleaching, and despite the significant interaction among factors in most occasions, the overall tendency pointed towards its increase (negative variations in $\mathrm{EC}_{50}$ values), showing also to be independent from extract type or plant species. In what concerns TBARS assay, and despite the significant interaction among effects

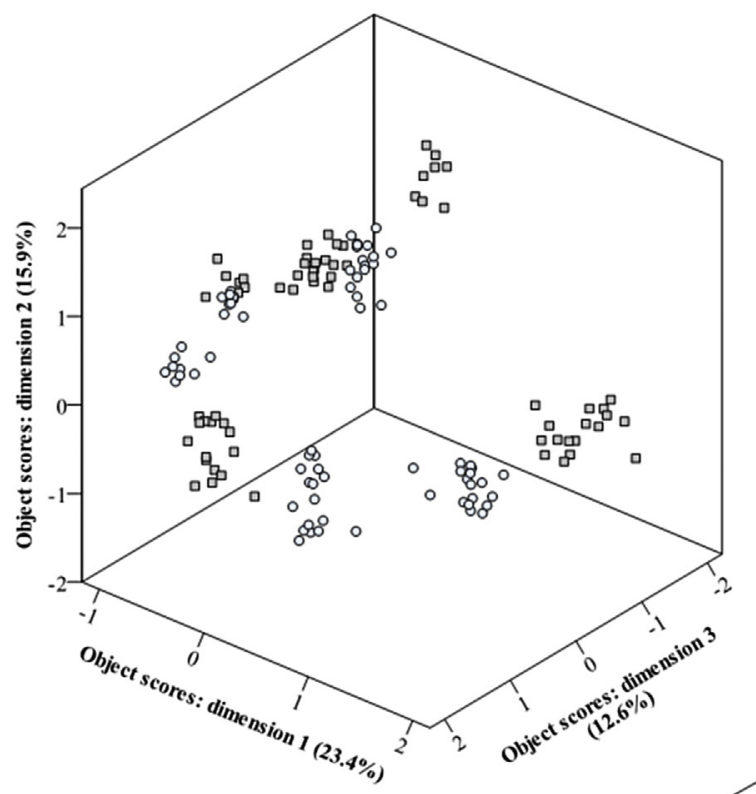

a)

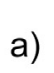

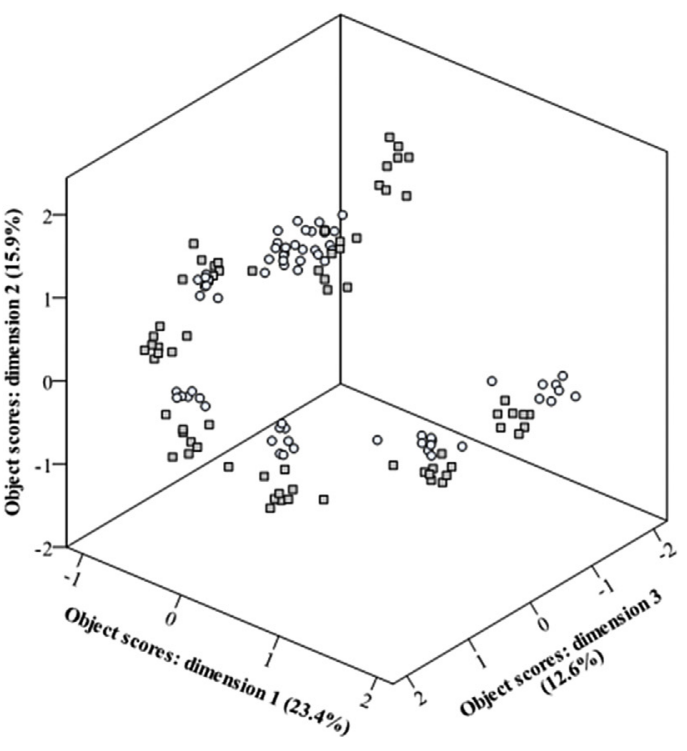

b)

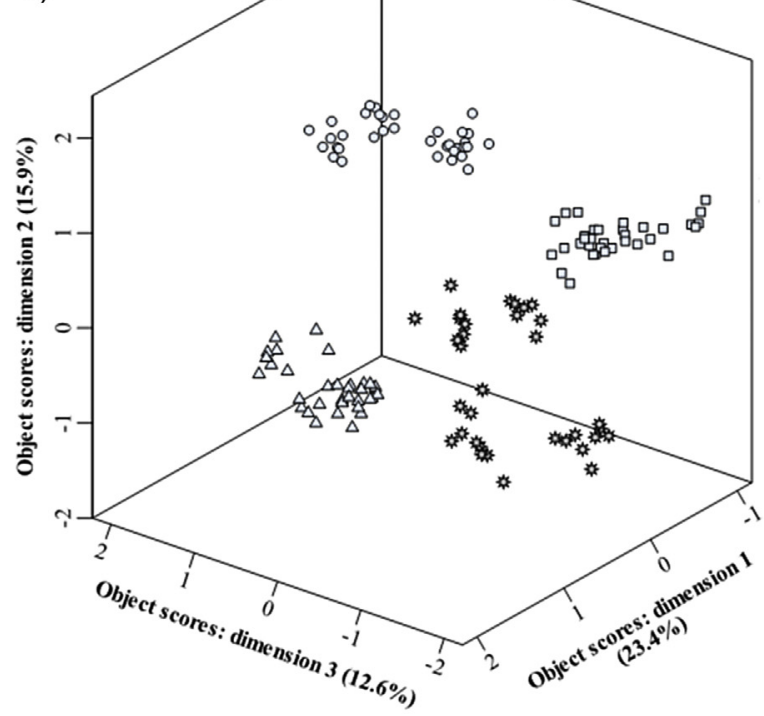

C)

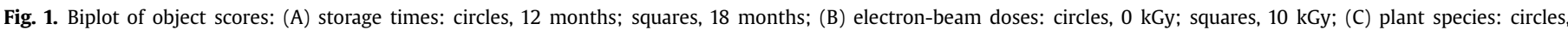

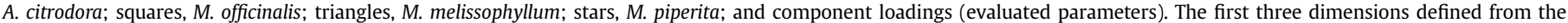

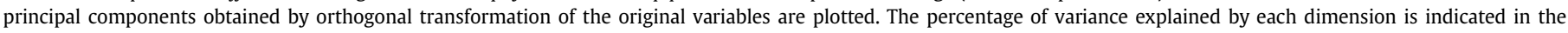
corresponding axis. 
(particularly for infusions), all species (except M. mellissophyllum) tended to present less activity throughout ST.

Concerning the contents in phenols and flavonoids, the interactions among ST and EB were also significant in most occasions. Still, some overall trends were identified, namely the higher amount of phenolic compounds in the methanolic extracts of irradiated $M$. officinalis and M. piperita and in both extracts of nonirradiated $A$. citrodora. The levels of flavonoids did not show to be significantly different among unirradiated and irradiated samples (except for the methanolic extracts of A. citrodora, M. officinalis and M. piperita). Additionally, and exempting M. mellissophyllum, total phenolic compounds and flavonoids levels tended to be higher in samples stored during 12 months, in comparison to the observed among those stored during 18 months.

\subsection{Principal component analysis (PCA)}

For the effects of ST and EB on several chemical and antioxidant parameters, some significant changes were identified, but it would also be interesting to find the parameters with the most noticeable changes within each studied factor, when considering differences of all assayed parameters simultaneously. Accordingly, the results were evaluated considering data for all studied EB doses, ST and plant species through a categorical principal components analysis (CATPCA).

The plot of object scores for different ST and EB (Fig. 1A, Fig. 1B), in which the first three dimensions are represented (first: Cronbach's $\alpha=0.942$; eigenvalue $=13.346$; second: Cronbach's $\alpha=0.906$; eigenvalue $=9.080$; third: Cronbach's $\alpha=0.877$; eigenvalue $=7.202$ ) account for most variance of all quantified variables ( $23.4 \%, 15.9 \%$ and $12.6 \%$, respectively). As it can be seen, markers corresponding to each factor level (12 months and 18 months, for ST, or $0 \mathrm{kGy}$ and $10 \mathrm{kGy}$, for EB) were not clustered individually. Therefore, there is no logic in proceeding with the analysis of associations among principal components and parameters.

On other hand, when comparing the effects of EB and ST as a function of plant species, the differences are obvious. Three main groups were formed: one corresponding to $A$. citrodora and $M$. officinalis, and two other groups, corresponding to each of the remaining species. According to the correlations among parameters and principal components (data not shown to avoid the overlapping of markers), A. citrodora and $M$. officinalis were mainly characterized by increases in protein content, malic acid, C18:0, C18:1n9, C23:0, MUFA, $\beta$-tocopherol and also in TBARS formation inhibition $\mathrm{EC}_{50}$ values (for either aqueous and methanolic extracts). Conversely, these same species had high decreases in fat, sucrose, trehalose, C20:0 and total tocopherols. As can be seen in Fig. 1C, markers corresponding to $M$. melissophyllum were localized in a diametrically opposite position; thereby, it is easily to conclude that the most representative changes in this species are exactly the opposite of those indicated for A. citrodora and M. officinalis. Finally, $M$. piperita was mainly characterized for presenting high increases in $\alpha$-tocopherol, C8:0, C15:1 and in $\mathrm{EC}_{50}$ values of DPPH scavenging activity (independently of extract type), reducing power (in methanolic extracts) and $\beta$-carotene bleaching inhibition (in methanolic extracts, also); on the other hand, these samples showed a significant decrease in glucose, total sugars, C6:0, C13:0 and C17:0.

\section{Conclusion}

In general, nutritional and colour parameters were the ones with less significant changes. The individual compounds, e.g. free sugars, organic acids, tocopherols and polyunsaturated fatty acids, revealed high reduction throughout storage, but the application of electron-beam, despite not preventing that reduction completely, had a significantly attenuating action. However, changes induced by irradiation and storage time showed some heterogeneity among species (particularly in M. melissophyllum). Therefore, further evaluation assays with different plant species are needed to achieve a better understanding of the effects resulting from electron-beam application.

\section{Acknowledgements}

The authors are grateful to the Ministry of Agriculture, Portugal, (Project PRODER/FEADER/EU $n^{\circ}$ 53514, AROMAP), for financial support of the work and E. Pereira grant, and to Foundation for Science and Technology (FCT, Portugal) and FEDER for financial support to CIMO (UID/AGR/00690/2013), J.C.M. Barreira (BPD/ 72802/2010) and L. Barros (SFRH/BPD/107855/2015) grants. Prof. A. Chmielewski, General Director of the Institute of Nuclear Chemistry and Technology, Warsaw, Poland, for allowing e-beam irradiations. The authors are also grateful to "MaisErvas - Aromáticas e Medicinais" for providing the plant material.

\section{References}

AOAC. (2002). Official methods of analysis (Vol. 16). Arlington VA, USA: Association of Official Analytical Chemists.

Barros, L., Pereira, E., Calhelha, R. C., Dueñas, M., Carvalho, A. M., Santos-Buelga, C. et al. (2013). Bioactivity and chemical characterization in hydrophilic and lipophilic compounds of Chenopodium ambrosioides L. Journal of Functional Foods, 5, 1732-1740.

Cabo Verde, S., Melo, R., Marcos, H., Silva, T., Nunes, I., Dores, V., et al. (2010). Radiation technology: Processes and products - concepts and applications (Vol. 1, pp. 5-21). India: Radiation Processing Technology Applications, Shriram Institute for Industrial Research.

Darfour, B., Agbenyegah, S., Ofosu, D. O., Okyere, A. A., \& Asare, I. K. (2014). Gamma irradiation of Tetrapleura tetraptera fruit as a post-harvest technique and its subsequent effect on some phytochemicals, free scavenging activity and physicochemical properties. Radiation Physics and Chemistry, 102, 153-158.

EU. (1999). Directive 1999/3/EC of the European Parliament and of the Council of 22 February 1999 on the establishment of a Community list of foods and food ingredients treated with ionising radiation. Official Journal of European Communities. L66/24-L66/25.

Farkas, J. (2006). Irradiation for better foods. Trends in Food Science \& Technology, 18 $1-5$.

Farkas, J. (2008). Irradiation as a method for decontaminating food - a review. International Journal of Food Microbiology, 44, 189-204.

Farkas, J., \& Mohácsi-Farkas, C. (2011). History and future of food irradiation. Trends in Food Science \& Technology, 20,1-6.

Haleem, R. M., Salem, M. Y., Fatahallah, F. A., \& Abdelfattah, L. E. (2014). Quality in the pharmaceutical industry - a literature review. Saudi Pharmaceutical Journal. http://dx.doi.org/10.1016/j.jsps.2013.11.004.

Hsu, W.-Y., Simonne, A., Jitareerat, P., \& Marshall, M. R., Jr. (2010). Low-dose irradiation improves microbial quality and shelf life of fresh mint (Mentha piperita L.) without compromising visual quality. Journal of Food Science, 75, M222-M230.

Hunter, C. (2000). Changing attitudes to irradiation throughout the food chain. Radiation Physics and Chemistry, 57, 239-243.

Jo, C., Son, J. H., Shin, M. G., \& Byun, M. W. (2003). Irradiation effects on color and functional properties persimmon (Diospyros kaki L. folium) leaf extract and licorice (Glycyrrhiza uralensis Fisher) root extract during storage. Radiation Physics and Chemistry, 67, 143-148.

Katusinn-Razěm, B., Novak, B., \& Razěm, D. (2001). Microbiological decontamination of botanical raw materials and corresponding pharmaceutical products by irradiation. Radiation Physics and Chemistry, 62, 261-275.

Migdal, W., \& Owczarczyk, B. (1998). The effect of ionizing radiation on microbiological decontamination of medical herbs and biologically active compounds. Radiation Physics and Chemistry, 52, 91-94.

Nagy, T. O., Solar, S., Sontag, G., \& Koenig, J. (2011). Identification of phenolic components in dried spices and influence of irradiation. Food Chemistry, 128, $530-534$.

Pereira, E., Antonio, A. L., Barreira, J. C. M., Barros, L., Bento, A., \& Ferreira, I. C. F. R. (2015b). Gamma irradiation as a practical alternative to preserve the chemical and bioactive wholesomeness of widely used aromatic plants. Food Research Internal, 67, 338-348.

Pereira, E., Antonio, A. L., Rafalski, A., Barreira, J. C. M., Barros, L., \& Ferreira, I. C. F. R. (2015a). Extending the use of irradiation to preserve chemical and bioactive properties of medicinal and aromatic plants: A case study with four species 
submitted to electron beam. Industrial Crops and Products, 77, 972-982.

Pereira, E., Barros, L. \& Ferreira, I. C. F. R. (2013). Chemical characterization of Ginkgo biloba L. and antioxidant properties of its extracts and dietary supplements. Industrial Crops and Products, 51, 244-248.

Roberts, P. B. (2014). Food irradiation is safe: Half a century of studies. Radiation Physics and Chemistry, 105, 78-82.
Warner, K., Miller, J., \& Demurin, Y. (2008). Oxidative stability of crude mid-oleic sunflower oils from seeds with $\gamma$ - and $\delta$-tocopherol levels. Journal of the American Chemical Society, 85, 529-533.

Wei, M., Zhou, L., Song, H., Yi, J., Wu, B., Li, Y., et al. (2014). Electron beam irradiation of sun-dried apricots for quality maintenance. Radiation Physics and Chemistry, 97, 126-133. 